\title{
Consultations with superstitious athletes in sport psychology
}

Volume 7 Issue 3 - 2017

\section{Short communication}

Superstition has been defined as "belief that, despite evidence to the contrary, certain behavior is causally related to certain outcomes.". Another definition describes superstition as "regularized, ritualistic actions performed to give people a sense of control and predictability in the face of challenges.". ${ }^{2}$ The role of superstitions in sports is often underestimated by outside observers and those not close to team or individual athletes. However, for those involved in sports such as coaches, athletes, and immersed fans, superstitions are many times treated as traditions that should be adhered to despite the amount of effort required or involved. Superstitions are more prevalent the higher the competitive level achieved and with an increased number of years being involved in sports. ${ }^{3}$

Compared to other areas of scholarly inquiry in the fields of sport sociology and sport psychology, research performed on superstitious behaviors in sport has been scant. In a previous study ${ }^{4}$ utilizing the Superstitious Ritual Questionnaire ${ }^{5} 208$ NCAA Division I athletes' superstitious behaviors and psychological characteristics were explored. In agreement with Todd \& Brown, ${ }^{6}$ superstitious behavior was not found to be affected by an athlete's locus of control. Also, male and female athletes differed somewhat in the types of superstitious habits. Other studies of superstitious behavior have investigated variables such as type of sport and certain personality characteristics (i.e., personal control, athletic identity, optimism/pessimism) as influential in subscribing to superstitious rituals.

Superstitions have been ingrained in the culture of the sporting world much longer than sport psychology specialists have been assisting athletes and teams. In many cases, superstitious behavior occurs before during and sometimes after the competitive event and are viewed as vital to good performance. Therefore, sport psychologists who ignore the importance of superstitions to sports participants are likely making an error in judgment. Adherence, or lack thereof, to superstitions may have an impact on maintaining or disrupting team chemistry.

Sport psychology consultants must face the dilemma of whether to perpetuate the seemingly irrational beliefs, or help coaches, athletes, and spectators become less dependent on, or remove superstitious behavior. While generally all consultations with sports participants are different, one variable to consider is the reasons athletes may choose to believe superstitions. First, many athletes learn superstitions from other coaches and athletes. ${ }^{7}$ Therefore as part of being involved in a team or individual sport, the ritualistic behavior becomes commonly accepted within a team. Even athletes who generally do not put much credence in superstitious beliefs, may begin cooperate so as not to seem different, disrupt the team, or simply due to peer pressure. ${ }^{8}$ However, an important consideration must be given to what is likely the most common reason superstitions are utilized. Superstitions are often used by fans, athletes, and coaches to attempt to control performance and outcomes in an atmosphere that has many unknowns

\author{
Kevin L Burke \\ Kinesiology Department, Queens University of Charlotte, USA
}

Correspondence: Kevin L Burke, Kinesiology Department, Queens University of Charlotte, 1900 Selwyn Avenue, Charlotte, North Carolina 28274, USA, Email kevinlburke@gmail.com

Received: October 28, 2016 | Published: February 10, 2017 and is unpredictable. For many sports participants, along with physical and mental practice, performing superstitions are part of what they believe helps them, or their team, prepare to play their best.

It is important to distinguish between pre-performance routines and superstitions. Pre-performance routines are actions generally taken just before a player performs a specific skill. For example, a basketball player at the free throw line may always dribble the basketball three times, take a deep breath, then attempt the free throw. Actions such as these generally are not superstitious behavior, but are performed to get the athlete physically and mentally ready to perform. ${ }^{9,10}$ Superstitions usually offer no logical progression to assist the performance of sports skills. The other distinguishing factor between pre-performance routines and superstitions is the sense of control. Coaches and athletes are in control of pre-performance routines - meaning they can alter and change routines to suit their purpose without much mental anguish in doing so. In most situations, superstitious traditions are not easily changed. However, sports fans, athletes, and coaches usually lack these same feelings of control when performing superstitious behaviors. In other words, superstitious beliefs may eventually seem to control the person involved. Also, coaches, athletes, and fans become extremely uncomfortable if unable to perform the ritualistic behaviors. Hence, many will experience extreme anxiety, discomfort, or become distracted if prevented from cooperating with a superstition.

Even though some sport psychologists may believe superstitions are irrational although it would be interesting to investigate the prevalence of superstitious behaviors among sport psychologists, to attempt to force superstitious subscribers to overcome the ritualistic practices is unnecessary. Even on those occasions when the sport psychology consultant believes the coach or athlete may benefit from becoming less reliant upon superstitious behaviors, offering more than a polite suggestion to work on this area may be extremely disconcerting for the individual or team. However, as is usually the case with clients who are seeking change, if an athlete requests help with becoming less dependent upon superstitions, certainly a sport psychology consultant may appropriately help the athlete reach this goal. 
Sport psychology consultants have personal beliefs and opinions that may be significantly different from their clientele. As with other aspects of counseling and consulting work, it is important to prevent personal biases from intervening with quality assistance provided to the client. Unless the client seeks assistance in doing so, sport psychology consultants should not insist sports fans, coaches, and athletes dispense of their beliefs in superstitions.

\section{Acknowledgments}

None.

\section{Conflicts of interest}

Author declares there are no conflicts of interest.

\section{Funding}

None.

\section{References}

1. Moran A. Attention training. In: Eklund \& Tenenbaum (Eds.), Encyclopedia of Sport and Exercise Psychology. Los Angeles: Sage, California, USA. 2014.

2. Coakley J. Sports in Society: Issues and Controversies (11th edn) McGraw-Hill, NY, USA. 2015.
3. Sage GH, Eitzen DS. Sociology of North American Sports (10th edn). Oxford University Press, NY, USA. 2016.

4. Burke KL, Czech DR, Knight JL, et al. An exploratory investigation of superstition, personal control, optimism and pessimism in NCAA Division I intercollegiate student-athletes.AthleticInsight: The Online Journal of Sport Psychology. 2006;8(2).

5. Bleak JL, Frederick CM. Superstitious behavior in sport: Levels of effectiveness and determinants of use in three collegiate sports. Journal of Sport Behavior. 1998;21(1):1-15.

6. Todd M, Brown C. Characteristics associated with superstitious behavior in track and field athletes: Are there NCAA divisional level differences? Journal of Sport Behavior. 2003;26(2):168-187.

7. Burke KL. Why athletes have such strange superstitions. Sporting News, USA. 2016.

8. Burke KL. How Cubs, Indians should handle their curses and superstitions in World Series. Sporting News, USA. 2016.

9. Burke KL, Brown D. Sport Psychology Library: Basketball. Morgantown, Fitness Information Technology, West Virginia, USA. 2003

10. Van Raalte JL, Brewer BW. Exploring Sport and Exercise Psychology (3rd edn). American Psychological Association, Washington, D. C., USA. 2014. 